\title{
Perfil demográfico e de mortalidade infantil do programa "Bom Começo", Medellín 2009-2016
}

\author{
Perfil socio demográfico y de mortalidad infantil programa "Buen Comienzo", Medellín \\ 2009-2016
}

\section{Sociodemographic and infant mortality profile of the program "Good Start", Medellin 2009-2016}

\author{
Grey Yuliet Ceballos-García* \\ Claudia Patricia Lopera-Arrubla** \\ Ángela Susana Lopera-Escobar***
}

\begin{abstract}
Autor de correspondencia
* $\triangle$ Enfermera, Magister en Salud Colectiva. Docente e Investigadora. Docente Ocasional de la Facultad de Enfermería de la Universidad de Antioquia. Correo: grey.ceballos@ udea.edu.co. Orcid: http://orcid.org/00000002-1146-1457. Medellín, Colombia.

*** Enfermera, Magister en Educación. Docente e Investigadora. Jefe del Centro de Investigación. Facultad de Enfermería de la Universidad de Antioquia. Correo: claudia.lopera@ udea.edu.co. Orcid: https://orcid.org/00000001-6637-2315. Medellín, Colombia.

**** Enfermera. Enfermera asistencial Clínica Sagrado Corazón. Correo: susana.lopera@ udea.edu.co Orcid: https://orcid.org/00000003-2745-4080. Medellín, Colombia.
\end{abstract}

(C) Universidad Francisco de Paula Santander. Este es un artículo bajo la licencia CC BY (https://creativecommons.org/ licenses/by/4.0/) (c) (1)

\section{Resumo}

Introdução: O Objetivo de Desenvolvimento Sustentável número três têm como propósito por fim às mortes evitáveis em recém nascidos e menores de 5 anos. Uma das estratégias que pode contribuir ao logro do mesmo é o programa "Bom Começo" Objetivo: Examinar variáveis sociais, demográficas e de mortalidade infantil das crianças menores de um ano que participaram do programa "Bom Começo" da cidade de Medellín no período 2009 a 2016. Materiais e métodos: Estudo quantitativo, descritivo, transversal, empregando bancos de dados secundários do município de Medellín. Foram calculadas frequências absolutas e relativas das variáveis, além disso a ração de prevalência e indicadores de mortalidade infantil e neonatal para Medellín. Analisaram-se 48.344 registros. Resultados: A ausência de afiliação das crianças ao sistema de saúde passou do $22.1 \%$ em 2009 a 4.4\% em 2016. Mais do 50\% dos participantes não estavam inscritos em programas de atenção primária à saúde de controle de crescimento e desenvolvimento. No período morreram 42 menores, 59\% do sexo masculino. As principais causas de morte foram as malformações congênitas, deformidades e anormalidades cromossômicas (23.8\%), enfermidades do sistema respiratório (19\%), doenças infecciosas e parasitarias (7.3\%). Conclusão: O coeficiente de mortalidade infantil do programa "Bom Começo" foi menor, comparado com a taça de mortalidade infantil para Medellín. A mortalidade infantil é resultado de diversos determinantes e as ações necessárias para salvar suas vidas são conhecidas. O desafio que segue se tendo é transferir o já conhecido à ação.

Palavras-chave: Política pública, saúde da criança, demografia, cuidado da criança.

\section{Resumen}

Introducción: El Objetivo de Desarrollo Sostenible número 3 tiene entre sus metas poner fin a las muertes evitables de recién nacidos y menores de 5 años. Una de las estrategias que puede aportar al cumplimiento de esta meta es el programa "Buen Comienzo". Objetivo: Examinar variables sociodemográficas y de mortalidad infantil de los niños menores de un año que participaron del programa Buen Comienzo del Municipio de Medellín en el periodo de 2009 a 2016. Materiales y métodos: Estu- 
dio cuantitativo descriptivo de corte trasversal, utilizando base de datos secundarias del municipio de Medellín. Se calcularon frecuencias absolutas y relativas de las variables, además de la razón de prevalencia e indicadores de mortalidad infantil y neonatal para Medellín. Se analizaron 48.344 registros. Resultados: la no afiliación de los niños al sistema de salud pasó de 22,1 \% en 2009 a 4,4 $\%$ en 2016. Más del $50 \%$ de los participantes no estaban inscritos en el programa de Crecimiento y Desarrollo. En el periodo murieron 42 menores, el $59 \%$ eran del sexo masculino. Las principales causas de muerte fueron las malformaciones congénitas, deformaciones y anomalías cromosómicas $(23,8 \%)$, enfermedades del sistema respiratorio $(19 \%)$, enfermedades infecciosas y parasitarias (7,3\%). Conclusión: el coeficiente de mortalidad infantil del programa Buen Comienzo fue menor, comparado con la tasa de mortalidad infantil para Medellín. La mortalidad infantil es el resultado de una compleja red de determinantes y las acciones necesarias para salvar sus vidas son conocidas. El desafío que se sigue teniendo es trasferir lo que ya se sabe a la acción.

Palabras clave: Política pública, salud infantil, demografía, cuidado al niño
E-ISSN 2322-7028

Vol. 17 No. 1

Ene - Abr 2020

Cúcuta, Colombia

\begin{abstract}
Introduction: The Objective of Sustainable Development number 3 has among its targets to put an end to the preventable deaths of newborns and children under the age of 5. One of the strategies that can contribute to this target is the program "Good Start". Objective: Examine the sociodemographic and infant mortality variables on children under the age of one who participated in the Good Start program from the municipality of Medellin during the period from 2009 to 2016. Materials and methods: Quantitative descriptive, cross-sectional study, using secondary data bases from the municipality of Medellin. Absolute and relative frequencies of the variables were calculated, as well as the reasons of prevalence, and infant and newborn mortality indicators in Medellin. 48.344 records were analyzed. Results: Children not having health insurance went from $22,1 \%$ in 2009 to $4,4 \%$ in 2016. More than $50 \%$ of the participants were not subscribed to the program Growth and Development. During this period, 42 minors died, $59 \%$ were males. The main causes of death were congenital malformations, deformations and chromosomal abnormalities $(23.8 \%)$, respiratory diseases (29\%), infection and parasitic diseases (7,3\%). Conclusion: The infant mortality coefficient of the program Good Start was lower, compared to the infant mortality rate in Medellin. Infant mortality is the result of a complex network of determinants, and the necessary actions to save their lives are known. The challenge that prevails is to transfer knowledge into action.
\end{abstract}

Keywords: Public policy, child health, demography, child care

\section{Introdução}

Segundo a Organização Mundial da Saúde (OMS) (1), a taxa mundial de mortalidade em crianças menores de 5 anos tem se reduzido mais da metade desde 1990, diminuindo de 93 a 41 mortes por cada 1.000 nascidos vivos entre 1990-2016; entretanto, segundo esta mesma organização, embora os avanços substanciais, ainda podem ser salvas muitas crianças da morte por causas previsíveis. A OMS (2) no ano de 2017, estabeleceu que 5.4 milhões de crianças menores de 5 anos morreram no mundo, sendo que $83 \%$ delas foram causadas por infecções, condições neonatais e nutricionais. De acordo com o Programa das Nações Unidas para o Desenvolvimento (3), entre os Objetivos de Desenvolvimento Sustentável (ODS) que começaram a reger em janeiro de 2016, o objetivo número 3 referente à saúde e bem-estar, estabelece como meta por o fim às mortes evitáveis nos recém nascidos e menores de 5 anos até o 2030.
Pode-se dizer, que a mortalidade infantil é um indicador sensível das condições de saúde de um país e faz referência ao número de mortes de crianças menores de um ano em determinado período de tempo (4).

Um estudo prévio realizado por Cardona e Cols. (5) determinou que a Colômbia presentou das taxas de mortalidade infantil mais baixas da América Latina de 2008-2010, cumprindo com a meta dos Objetivos de Desenvolvimento do Milênio na redução da mortalidade infantil, atingindo 16.8 mortes em menores de 1 ano por cada 1000 nascidos vivos para 2016. Segundo o Departamento Administrativo Nacional de Estatísticas (DANE) (6), as diferenças regionais são dramáticas, embora existam estados que reportaram taxas de mortalidade infantil superiores de $30 \mathrm{em}$ 2016, o estado de Antioquia teve em 2015 uma taxa de mortalidade infantil de 8.5 e na cidade de Medellín foi de 8.1 (7). 
ISSN-PRINT

1794-9831

E-ISSN 2322-7028

Vol. 17 No. 1

Ene - Abr 2020

Cúcuta, Colombia
Cabe-se frisar que os fatores de risco e fatores protetores para a mortalidade infantil, tem sido amplamente documentada. O estudo realizado pelo Instituto Nacional de Saúde (INS) da Colômbia (8) demonstrou que a mortalidade em menores de um ano esteve associada significativamente com o: sexo da criança, o ensino da mãe, a idade gestacional ao nascimento, o aleitamento materno e o número de moradores do lar. Da mesma maneira, Jaramillo e Cols. (9) sugeriram que as variáveis: peso ao nascimento, acesso ao acompanhamento pré-natal, o ensino da mãe, acesso aos serviços de saúde durante o atendimento do parto e pós-parto estão relacionadas com a maior supervivência das crianças.

As políticas públicas respondem às necessidades da população e definem como são distribuídas as responsabilidades e recursos entre os atores sociais (10). Na Colômbia, existem políticas que procuram proteger a primeira infância, entre elas: o programa do "zer0 a 5empre" formulado em 2011 pelo governo nacional e que teve as suas origens na cidade de Medellín com o programa "Bom Começo".

Conforme a Prefeitura de Medellín (11-14), o programa "Bom Começo" promove o desenvolvimento de crianças menores de 6 anos, priorizando o atendimento às famílias em maiores condições de vulnerabilidade da cidade. Para lograr os seus objetivos articula o trabalho de diversas áreas do governo local: a secretaria de inclusão social e família, a secretaria da educação, a secretaria da saúde e o Instituto de Deportes e Recreação de Medellín (INDER); aliados ainda com o Instituto Colombiano de Bem-estar Familiar (ICBF), o Ministério da Educação Nacional e a empresa privada. Neste sentido, o acompanhamento dessas crianças inclui intervenção profissional de nutrição, psicologia, educação física e pedagogia, além de suplementação nutricional.

Analisar o efeito desse tipo de políticas sobre as condições de vida e saúde das pessoas, é imperativo dos governos e sociedade. No caso do "Bom Começo, Cardoso $\&$ Medina (15), evidenciaram benefícios como o incremento do peso corporal nas crianças que faziam parte do programa. As descrições feitas por Quintero e Cols. (16) demostraram que as crianças que participam do programa mudaram favoravelmente os seus hábitos alimentares favorecendo a sua saúde e o estado nutricional.

Deve-se destacar que o mundo continua em dívida com a saúde das crianças, pelo que reduzir a mortalidade infantil continua sendo um dos ODS, sabendo-se que, a maioria das mortes são causadas pelas condições injustas e evitáveis. Neste sentido, examinar o perfil demográfico da população beneficiada do programa "Bom Começo" em Medellín poderá contribuir no estabelecimento do potencial do mesmo na redução da mortalidade infantil, fornecendo elementos e informação valiosa para a toma de decisões em saúde, assim como a implementação de políticas que procurem melhorar as condições com que nascem e se desenvolvem as crianças, e assim, favorecer a diminuição de inequidades em saúde da população.

\section{Objetivos}

\section{Objetivo General}

Examinar as variáveis sociais, demográficas e de mortalidade infantil das crianças menores de um ano que participaram do programa "Bom Começo" da cidade de Medellín no período de 2009-2016.

\section{Objetivos Específicos}

- Descrever as variáveis sociais e demográficas das crianças que participaram do programa "Bom Começo

- Identificar o comportamento e as causas de mortalidade infantil das crianças menores de um ano que faziam parte do programa "Bom Começo

\section{Materiais e Métodos}

Estudo quantitativo, descritivo, transversal, empregando os bancos de dados do programa "Bom Começo" fornecidos pela Secretaria da Educação da cidade de Medellín do período de 2009-2016. Os dados de mortalidade infantil e segurança social foram subministrados pela Unidade de Gestão de Informação e Conhecimento da Secretaria de Saúde de Medellín. Foram selecionados todos os registros de crianças menores de um ano e residentes de Medellín. Obtiveram-se 48.344 registros que foram analisados.

A informação foi inicialmente registrada no programa Microsoft Excel, posteriormente exportou-se e analisouse no software SPSS versão 23. Calcularam-se frequências absolutas e relativas das variáveis: idade (quantificada em meses), sexo, tipo de afiliação ao sistema de saúde (contributivo, subsidiado, especial ou população pobre não assegurada -PPNA), participação do programa de crescimento e desenvolvimento (sim/não), cuidador principal (mãe, pai, outro), nível de ensino e ocupação 
dos pais. Obtiveram-se as taxas de mortalidade infantil e neonatal para a cidade de Medellín para o ano 2000 e, o coeficiente de mortalidade específica para as crianças que participaram no "Bom Começo". Essas taxas foram construídas a partir dos dados registrados no DANE no website de libre acesso de estatísticas vitais. Os dados foram processados com a ajuda da unidade de Gestão da Informação e Conhecimento da Secretaria de Saúde de Medellín. O cálculo da taxa de mortalidade neonatal se obteve a partir do ano 2005. A Construção do coeficiente específico de mortalidade para as crianças que faziam parte do programa "Bom Começo" logrou-se calculando o número de participantes por ano, sob o total das crianças que morreram no mesmo período.

Este estudo contou com a aprovação do comité de ética da Faculdade de Enfermagem da Universidade de Antioquia e a licença de uso dos registros da Secretaria da Educação de Medellín; não se estabeleceu contato direto com as crianças ou as suas famílias. A confidencialidade da informação durante todo o processo foi mantida. A pesquisa realizada está classificada como um estudo "sem risco", de acordo com a Resolução 8430 de 1993 do Ministério da Saúde (17), que faz referencia as normas científicas, técnicas e administrativas para a pes- quisa em saúde, e cumpre com os diretrizes bioéticas de Beauchamp e Childress de respeito, justiça e beneficência que são expostos na mesma Resolução.

\section{Resultados}

\section{Características sociais e demográficas}

A distribuição por sexo esteve próxima do $50 \%$. Sobre o tipo de afiliação ao sistema de saúde, as crianças afiliadas no regime contributivo incrementaram-se, passando do $8.1 \%$ em 2009 a 41.4\% em 2016; no regime contributivo , passou do 32.8\% em 2009 a 40.7\% em 2016. A população pobre não assegurada (PPNA) teve uma redução progressiva ano a ano, passando do $22.1 \%$ em 2009 ao $4,4 \%$ em 2016. Chamou a atenção que muitas das crianças dos registros do "Bom Começo" não foram encontradas nos bancos de dados de assegurados da Secretaria de Saúde; entretanto, este registro melhorou durante o período de medição, de $37 \%$ em 2009 reduzindo-se progressivamente até o 13.5\% em 2006 (Figura 1). Em 2010 o $13.7 \%$ dos menores de um ano que participaram do programa, foram vítimas do conflito armado (afastamento forçado das suas terras), estas cifras também diminuíram até o 1.2\% registrado em 2016.

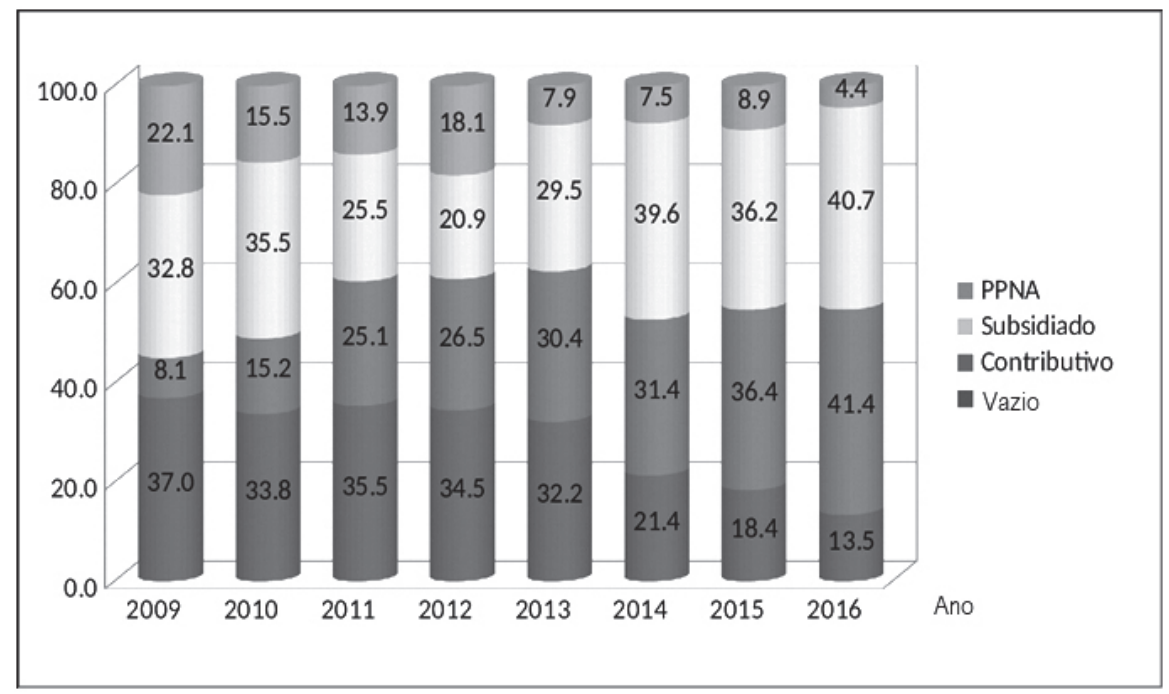

Figura 1: Regime de afiliação ao Sistema de Seguridade Social em Saúde, 2009-2016 Fonte: Unidade de Gestão da Informação e do Conhecimento da Secretaria de Saúde de Medellín.

A responsabilidade do cuidado das crianças, recaiu principalmente sob as mães em $94 \%$ dos casos. O grau de ensino da mesma, só reportou informação no período de 2014-2016. Os estudos de segundo grau completo e incompleto representaram o $65 \%$ da população; obser- vou-se que o nível de ensino segundo grau completo do cuidador passou do $35.5 \%$ em 2014 a 36.7\% em 2016 além da redução do segundo grau incompleto do $37.8 \%$ em 2014 a 33.2\% em 2016. Os cuidadores com formação técnica ou tecnológica passaram de 11.5\% em 2014 a 
ISSN-PRINT

1794-9831

E-ISSN 2322-7028

Vol. 17 No. 1

Ene - Abr 2020

Cúcuta, Colombia
15.8\% em 2016. A ocupação delas era do lar no $68 \%$; em 2014 15.5\% sua ocupação era ser estudante, já em 2016 foi de $13.8 \%$.

Mortalidade infantil em crianças menores de um ano do programa "Bom Começo"

Do total de crianças menores de 1 ano no período de medição, 42 morreram, com maior número de mortes nos anos 2014 e 2015, 28,6\% para cada ano (Tabla 1). A distribuição relativa segundo a idade da morte foi do $71.4 \%$ antes de completar 7 meses de idade, observando-se que aos dois $(16.7 \%)$, cinco $(14,3 \%)$ e seis $(19,0 \%)$ meses foram as idades que mais se apresentaram este desfecho.

O 53,7\% das crianças que morreram pertenciam ao regime contributivo, o 2,4\% ao PPNA e 9,8\% não tinha registro nessa variável. A ração de prevalência (RP) entre a mortalidade infantil e pertencer ao regime subsidiado foi de 1,6 (IC95\% 0.8-3.1) (Tabela 1).

Referente ao lugar de moradia, encontrou-se que a maioria morava no setor Popular da Comuna 1, seguido do bairro San Javier, Manrique, Santa Cruz e Villa Hermosa. A distribuição por lugar

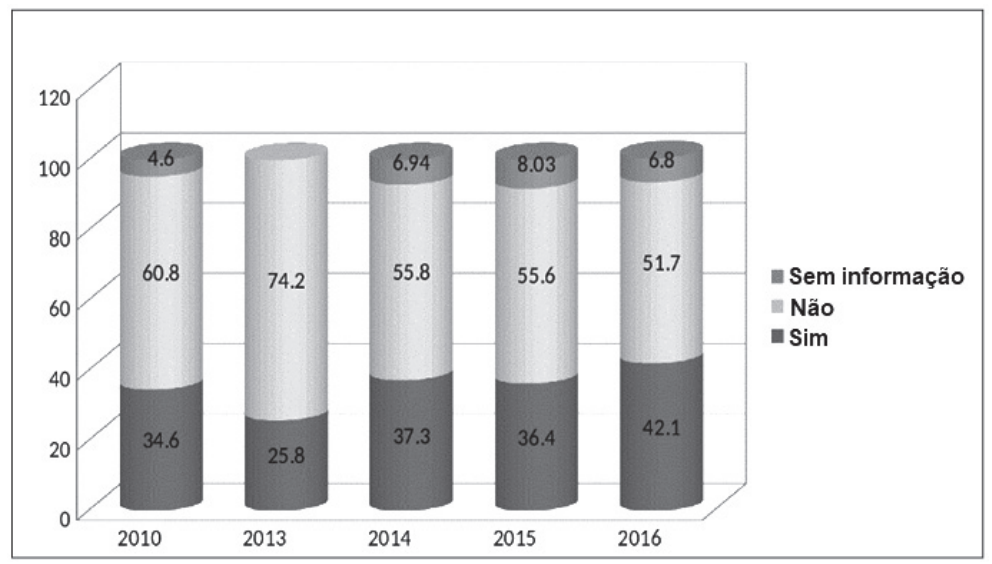

Figura 2: Participação em programas de Crescimento e Desenvolvimento, 2010-2016

Fonte: Bancos de dados do programa "Bom Começo" e Secretaria de Ensino de Medellín.

de residência das crianças que faleceram foi semelhante à da população geral: 14,3\% no bairro Santa Cruz; 11,9\% Popular, Manrique com 11,9\%; Aranjuez e San Javier com 9,5\%; respectivamente. Cabe destacar que do setor rural de Santa Elena no ano 2014 participaram do programa 28 crianças, das que morreram 2. A maioria dos participantes
(55\%) não estavam comparecendo a nenhum programa de crescimento e desenvolvimento (figura 2). Estes dados foram semelhantes nas crianças que faleceram. Ao realizar a análise de mortalidade infantil e pertencer ao programa de crescimento e desenvolvimento evidenciou um RP de 1,4 (IC 95\% 0,7-2,9) (Tabela 1). 
Tabela 1. Características demográficas e de saúde de crianças menores de 1 ano que fizeram parte do programa "Bom Começo", 2009-2016

\begin{tabular}{|c|c|c|c|c|}
\hline \multirow[t]{2}{*}{ Variable } & \multirow{2}{*}{$\begin{array}{l}\text { Total población menor de } 1 \text { año \% } \\
\text { Variável }\end{array}$} & \multicolumn{2}{|c|}{ Menores que fallecieron en el periodo $\%$} & \multirow{2}{*}{$\begin{array}{c}\text { RP (IC 95\%) } \\
\text { Ração de Prevalença } \\
\text { (IC95\%) }\end{array}$} \\
\hline & & $\begin{array}{c}\text { Total população menor de } \\
1 \text { ano (\%) }\end{array}$ & $\begin{array}{l}\text { Menores que faleceram no } \\
\text { período (\%) }\end{array}$ & \\
\hline \multicolumn{5}{|l|}{ Sexo } \\
\hline Masculino & & 49,9 & 59,0 & $1.3(0.8-2.4)$ \\
\hline Femenino & & 50,1 & 41,0 & \\
\hline \multicolumn{5}{|c|}{ Tipo de afiliação ao sistema de Saúde (SGSSS)* } \\
\hline Contributiv & & 27,9 & 34,1 & \\
\hline Subsidiado & & 32,9 & 53,7 & $1.6(0.8-3.1)$ \\
\hline População I & pre Não Assegurada & 11,8 & 2,4 & \\
\hline Sem Inform & & 27,4 & 9,8 & \\
\hline \multicolumn{5}{|c|}{ Participação no programa de crescimento e desenvolvimento } \\
\hline Sim & & 33,9 & 34,1 & \\
\hline Não & & 60,3 & 48,8 & $1.4(0.7-2.9)$ \\
\hline Sem Inform & & 5,8 & 17,1 & \\
\hline \multicolumn{5}{|c|}{ Esquema de vacinação } \\
\hline Completo & & 90,5 & 78,6 & \\
\hline Incompleto & & 3,2 & 2,4 & $* *$ \\
\hline Sem inform & & 6,3 & 19 & \\
\hline \multicolumn{5}{|c|}{ População vítima do conflito armado } \\
\hline Não & & 93,8 & 97,6 & \\
\hline Filho de pai & etirado de grupo armado & 0,1 & 0 & \\
\hline Em situaçãc & e afastamento forçado & 4,5 & 2,4 & $* *$ \\
\hline Sem inform & & 1,1 & 0 & \\
\hline ** Não foi cal & & & & \\
\hline
\end{tabular}

1794-9831

E-ISSN 2322-7028

Vol. 17 No. 1

Ene - Abr 2020

Cúcuta, Colombia

Fonte: Bancos de dados do programa "Bom Começo", Secretaria de Ensino de Medellín.

*Sistema Geral de Seguridade Social em Saúde.

A figura 3 representa as taxas de mortalidade infantil (TMI) para Medellín, mortalidade neonatal e coeficiente de mortalidade infantil do programa "Bom começo". A TMI desde o ano 2000 tem evidenciado uma redução significativa cada ano, passando de 17 mortes para cada 1.000 nascidos vivos, a 8.1/ 1.000 no ano 2015. A taxa de mortalidade neonatal, também diminuiu, passando de 9.0/ 1.000 nascidos vivos em 2005 a $5.4 \mathrm{em} 2015$. Respeito ao coeficiente de mortalidade infantil das crianças do "Bom Começo" passou d0 0.2 em 2010 ao 0.8 em 2019; influenciado o acréscimo pela TMI de 1.6 / 1.000 menores de 1 ano participantes do programa. 
ISSN-PRINT

1794-9831

E-ISSN 2322-7028

Vol. 17 No. 1

Ene - Abr 2020

Cúcuta, Colombia

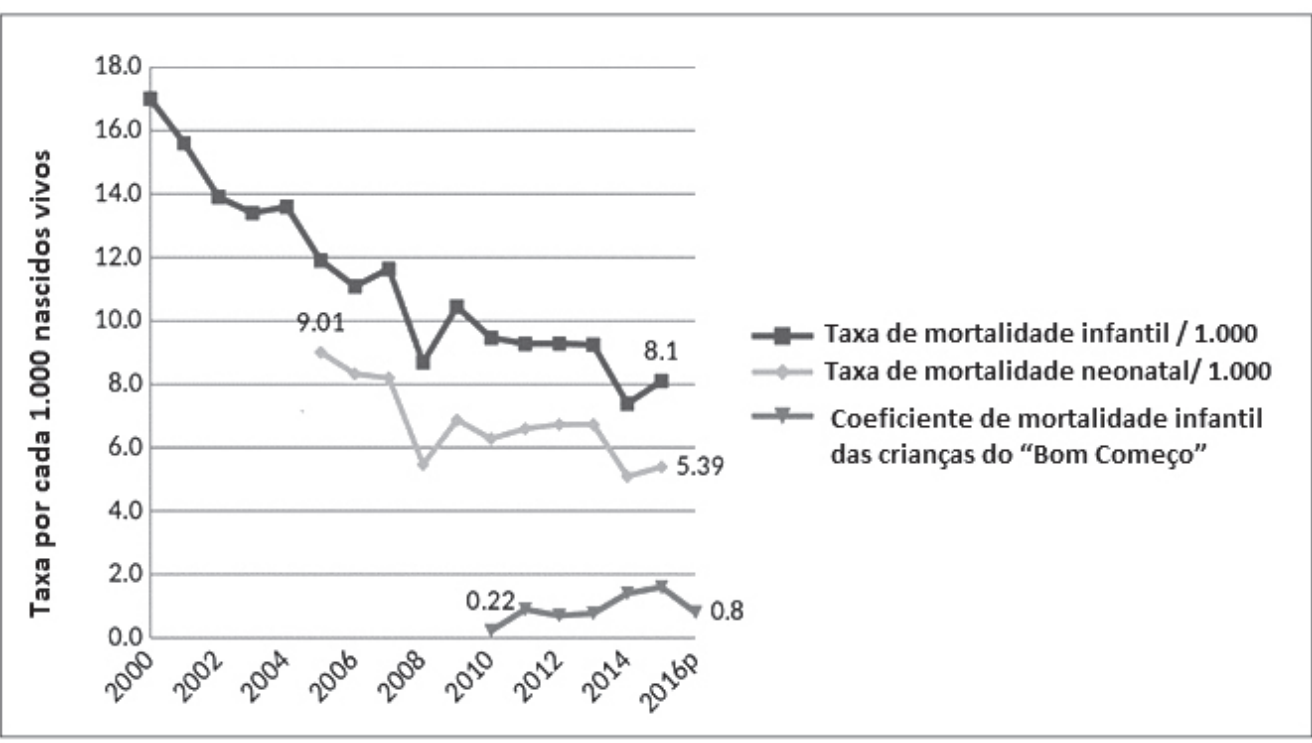

Figura 3: Mortalidade infantil em Medellín, 2000-2016

Fuente: DANE, Departamento Administrativo Nacional de Estadtstica. Processado pela Unidade de Gestão da Informação e o Conhecimento, Secretaria de Saúde de Medellín. P: Cifras preliminares.

Esclarecimento: Os valores de 2005-2011 correspondem à Secretaria de Saúde de Medellín. Isto dado que até 2011 tinha se implementado na Secretaria o processo da codificação das causas das mortes, a partir desse ano então o DANE é a fonte oficial. É importante mencionar que a mortalidade neonatal possui um sub-registro, pois as crianças devem fazer parte do programa para pesquisar nos registros.

As principais causas de morte nas crianças foram: as malformações congênitas, as deformidades e anomalias cromossômicas $(23,8 \%)$ : seguidas das doenças do sistema respiratório (19\%), doenças do sistema nervoso, órgãos dos sentidos e do sistema circulatório com $14,1 \%$ em cada uma. Ainda nessa população, as doenças infecciosas e parasitarias foram causa de morte em $7,1 \%$ dos casos (Tabela 2).

Tabla 2. Causas básicas de morte segundo grupos CIE-10 de menores de 1 ano que faziam parte do programa "Bom Começo", 2009-2016

\begin{tabular}{lcc}
\hline Grupo de causa básica de morte & $\mathrm{N}$ & $\%$ \\
\hline XVII Malformações congénitas, deformidades e anomalias cromossômicas & 10 & 23,8 \\
X Doenças do sistema respiratório & 8 & 19,0 \\
VI-VIII Doenças sistema nervoso e órgãos dos sentidos & 6 & 14,3 \\
IX Doenças do Sistema circulatório & 6 & 14,3 \\
I Doenças infecciosas e parasitarias & 3 & 7,1 \\
XI Doenças do sistema Digestivo & 2 & 4,8 \\
XVI Afetações originadas no período perinatal & 2 & 4,8 \\
X Causas externas de traumatismos e envenenamento & 2 & 4,8 \\
II Tumores & 1 & 2,4 \\
III Doenças sangre, órgãos hematopoiéticos e transtornos mec. Imunidade & 1 & 2,4 \\
XVIII Sintomas, signos e estados morbosos mal definidos & 1 & 2,4 \\
Total & 42 & 100,0 \\
\hline
\end{tabular}

Fonte: DANE, Departamento Administrativo Nacional de Estatística. Processado pela Unidade de Gestão da Informação e o Conhecimento, Secretaria de Saúde de Medellín. P: Cifras preliminares. 


\section{Discussão}

Este estudo demonstrou a redução progressiva ds taxas de mortalidade infantil e neonatal em Medellín, resultados semelhantes aos estudos prévios desenvolvidos por Jaramillo e col. (9), Maceira (18), e Quiroz e col. (19) nos que evidenciaram que as taxas de mortalidade têm se reduzido na Colômbia e no estado de Antioquia. Considerando o reportado pela Secretaria de Saúde e Proteção Social de Antioquia (17), entre 2005 e 2015 a mortalidade infantil manteve a tendência constante ao decesso, passando de 16,8 mortes / 1.000 nascidos vivos em 2005 a 10,9 em 2015, sendo Antioquia um dos estados com as taxas de mortalidade infantil mais baixas do país.

Sabendo-se que o programa não tem explicitamente estabelecido dento os seus objetivos a redução da mortalidade infantil, este estudo demonstrou através do coeficiente de mortalidade infantil para o "Bom Começo" indicadores ainda mais satisfatórios comparado com a TMI da cidade de Medellín; isto poderia ser explicado pelo acompanhamento realizado pelo programa nas áreas de: saúdes, nutrição, apoio psicossocial e ensino, o que tem concordância com outros estudos que apontaram os resultados do "Bom Começo" como positivos para as condições de saúde das crianças.

Por outro lado, Augsburger e col. (20) evidenciaram que as possibilidades das crianças sobreviverem ao primeiro ano de vida são dependentes das condições do espaço que moram as suas famílias, essa desigualdade de estratos de melhores ou piores condições de vida, impactam a mortalidade pós-neonatal. Segundo a OMS (1) as crianças malnutridas, especialmente as classificadas com mal nutrição aguda grave, têm maiores probabilidades de morrer por doenças comuns da infầncia como: diarreia, pneumonia e paludismo. Diversos fatores relacionados com a nutrição contribuíram aproximadamente em $45 \%$ das mortes de crianças menores de 5 anos. Quintero e col. (16) sinalaram como positivo o impacto do programa "Bom Começo" nos indicadores de peso e comprimento ao nascimento das crianças. Também encontraram que os menores que assistiram ao programa, mudaram favoravelmente os seus hábitos alimentares e adquiriram rutinas que melhoravam a sua saúde, sendo que as mães estenderam essas mudanças ao grupo familiar.

A Comissão Sobre os Determinantes Sociais da Saúde da OMS (21) demonstrou através de diversos estudos, como as condições de moradia das pessoas está relacionada diretamente com a sua saúde e esperança de vida.
Neste sentido, os governos são estimulados a trabalhar em diferentes líneas desde o econômico, político, administrativo a favor da educação, cultura, entre outros; e a primeira linha do chamado é dedicar maior atenção ao desenvolvimento das crianças e a sua educação.

O atual sistema de saúde colombiano, instituído através da lei 100 de 1993 (22), estabeleceu a afiliação obrigatória ao sistema por meio de sois regimes: o contributivo para as pessoas com capacidade de pagamento (trabalhadores dependentes e independentes, também os aposentados); e o regime subsidiado pelo governo para as pessoas sem capacidade de pagamento. Um terceiro regime é denominado Pessoa Pobre Não Assegurada (PPNA), que considera as pessoas que não pertencem a um u outro dos regimes, portanto não tem acesso aos serviços de saúde, só o atendimento de emergência, esperando-se que em algum momento ingressem ao sistema incorporando-se em algum dos regimes.

De acordo com a prefeitura de Medellín (12), o programa "Bom começo" procura favorecer à população em condições de maior vulnerabilidade da cidade, explicando a elevada porcentagem de crianças que participaram do programa que pertenciam ao regime subsidiado de afiliação em saúde ou estavam registrados como PPNA. Neste sentido, em 2009 o 22,1\% dos menores que faziam parte do programa, estavam como PPNA; entretanto, a partir de 2013 a prefeitura de Medellín incremento esforços para vincular a população ao sistema de saúde, demostrando uma redução da categoria PPNA em 2016.

Segundo Maceira (18), ao diminuir a porcentagem de PPNA se espera, neste caso, que as crianças posam ter maior acesso aos serviços de atenção básica e cuidado da saúde como: atenção pré-natal, acompanhamento do crescimento e desenvolvimento infantil, ações de promoção da saúde e prevenção de doenças; e, atenção por doenças gerais, mediante a garantia do acesso aos serviços de saúde através das Entidades Promotoras de Saúde (EPS). De estas entidades se espera a articulação das diferentes iniciativas, gerando um trabalho coletivo com estratégias de Atenção Integrada às Doenças Prevalentes na Infầncia (AIDPI), a Estratégia Instituições Amigas da Mulher e a Infância (IAMI) e o Programa Ampliado de Imunização (PAI).

Historicamente, os serviços cobertos pelos planes de beneficio do regime contributivo e subsidiado são diferentes, colocando em desvantagem às pessoas afiliadas no subsidiado. Neste estudo, a maioria dos meno- 
ISSN-PRINT

1794-9831

E-ISSN 2322-7028

Vol. 17 No. 1

Ene - Abr 2020

Cúcuta, Colombia res que morreram pertenciam ao regime subsidiado, coincidindo com o reportado por Quiroz e col. (19) e a Secretaria de Saúde e Proteção Social de Antioquia (23), donde observaram-se maiores taxas de mortalidade nas pessoas pertencentes ao subsidiado; concluindo que isto pode ser causado pelas inequidades no acesso e qualidade dos serviços de saúde. Por outro lado, Jaramillo e col. $(24,25)$ asseguraram que estar afiliado ao contributivo representa redução na mortalidade infantil pelo melhor acesso geográfico, a quantidade e a qualidade dos serviços de saúde, mesmo com melhor tecnologia. Respeito com o acesso geográfico, previamente Rojas \& Caicedo (26), reportaram que morar a uma distância maior de 5 quilômetros do centro de saúde está associado com risco de morte.

A recente pesquisa revela que a mãe é a principal cuidadora das crianças menores de um ano, com maiores níveis de ensino; neste sentido Jaramillo e col. (25) sugeriram que o aumento nos níveis de ensino nas mães está negativamente relacionado com a mortalidade infantil . Ezeh e col. (27) também reportaram que crianças que nasceram de mães que não tiveram acesso ao ensino tiveram maiores probabilidades de morrer. Segundo Leyton \& Valenzuela (28) a mãe no seu papel de cuidadora principal das crianças, é quem identifica o mal estar ou problema de saúde do seu filho e segundo a compreensão dos sintomas do seu filho toma a decisão de aplicar medidas caseiras ou acudir a um especialista.

Embora a distribuição por sexo encontrada foi homogênea, as maiores taxas de mortalidade apresentaram-se em crianças do sexo masculino; este achado foi previamente descrito por Quiroz e col. (19) que demostraram que as crianças do sexo masculino morrem em maior proporção que as do sexo feminino; assim também o Ministério da Saúde e a Proteção Social de Colômbia (21) reportou que as taxas de mortalidade nos meninos foi maior entre 2025\% no período de 2005-2016.

Por outro lado, as causas de óbito foram: as malformações congênitas, deformações e anomalias cromossômicas que foram a s principais causas; seguido das enfermidades do sistema respiratório e ainda se presentaram mortes por doenças infecciosas e parasitarias. Este perfil epidemiológico coincidiu com o reportado pelo Ministério da Saúde e a Proteção Social (29) que nos dados de 2005-2011 indicaram que as principais causas de morte infantil foram: algumas afecções do período perinatal, seguido das malformações congênitas, signos, sintomas e achados anormais clínicos e de laboratório não clas- sificados no terceiro lugar; em quarto lugar as doenças respiratórias e no quinto lugar as doenças e infeções parasitarias. Segundo a OMS (!) em 2015, mais da metade das mortes em crianças menores de 5 anos deveram-se a causas previsíveis e tratáveis mediante intervenções simples e acessíveis; neste sentido, o fortalecimento dos sistemas de saúde para que todas as crianças acedam a tais intervenções salvará a vida de muitas crianças.

O Instituto Nacional de Saúde da Colômbia (8) define a morte evitável, as mortes que, segundo os conhecimentos médicos atuais e a tecnologia, poderiam evitar-se pelo sistema de saúde através da prevenção e/ou tratamento. No perfil de mortalidade encontrado nesta pesquisa, chama a tenção que ainda se presentem mortes por condições evitáveis como as doenças do sistema respiratório, infecções parasitárias e algumas afecções do período perinatal. Neste ponto, Mogollon \& Garcia (30) indicaram que as medidas que pudessem ser tomadas para salvar a vida dessas crianças têm a ver com melhoras no acesso aos serviços de saúde, o diagnóstico precoce, o tratamento oportuno, as condições sociais e econômicas, de saneamento ambiental e educação sanitária.

De acordo com a Comissão econômicas para a América Latina e o Caribe-CEPAL (31), uma nutrição adequada contribuiu de maneira fundamental na realização do direito ao disfrute do mais alto nível possível de saúde física e mental das crianças; neste sentido, o programa "Bom Começo" têm realizado um importante trabalho por melhorar as condições nutricionais das gestantes e as crianças que participam do programa. Em tal sentido, Cardoso \& Medina (15), estudaram o impacto do programa "Bom Começo" nos indicadores de peso ao nascimento, mostrando benefícios no incremento do peso nas crianças que faziam parte do programa; já Quintero e col.(16) avaliaram o componente nutricional encontrando que as crianças que assistiram ao programa, mudaram favoravelmente os hábitos alimentares, favorecendo a sua saúde e estado nutricional.

Destaca-se a importância do programa Atenção Primaria à Saúde (APS) que está mais vigente que nunca antes, principalmente no relacionado ao cuidado da saúde infantil. Neste aspecto, Andrade e col. (32) concluíram que o trabalho de enfermagem dentro da APS é uma ferramenta fundamental, que exige cuidado ao entorno familiar, que esteja preparado para detectar situações de alarme nas crianças procurando uma ação cooperativa em rede a favor da saúde das crianças. 
Cabe-se destacar o esforço do governo local e nacional para evitar a morte infantil, isto se viu refletido na redução das taxas de mortalidade; entretanto, neste estudo demostrou-se que estas ações devem ser fortalecidas, com o objetivo de melhorar as condições nas que nascem e crescem as nossas crianças. Por tanto, é preciso compreender que a mortalidade infantil é consequência de uma complexa rede de determinantes em diversos níveis, que van desde as condições sociais, econômicas e políticas nas que nascem e crescem as crianças, passando pelas próprias condições biológicas e a atenção nos serviços de saúde $(21,33)$.

Definitivamente, o pessoal da saúde especialmente o de enfermagem, têm um papel fundamental na realização das ações que permitam as crianças incrementar o acesso aos serviços de promoção da saúde e prevenção da doença, em tratamentos oportunos e de qualidade; sempre, acompanhados das medidas tendentes a reduzir as inequidades em saúde, relacionadas com os determinantes sociais de saúde como acesso à água potável, saneamento e educação sanitária entre outros, de tal forma contribua não só à sobrevivência das crianças, também a viver dignamente. As intervenções necessárias para salvar a vida das crianças são amplamente conhecidas e o principal desafio que ainda se têm é transferir o conhecimento próprio da disciplina de enfermagem à ação.

\section{Limitaciones del estudio}

Pelo fato de ser um estudo que empregou bancos de dados sequndarios que obteve informaçao de diversas fontes u operadores do programa "Bom Começo", encontraram-se dificuldades na completitud da informação em algumas variáveis.

\section{Conclusões}

Embora a finalidade do programa "Bom Começo" não tenha o propósito da redução da mortalidade infantil, evidenciaram-se nos seus participantes, menores TMI comparadas com indicadores locais; isto poderia ser a causa do acompanhamento realizado pelo programa nas diferentes áreas: saúde, nutrição, apoio psicossocial e ensino, concordando com outros estudos que apontam os efeitos positivos do programa sob as condições de saúde das crianças. O programa "Bom Começo" prioriza as famílias mais vulneráveis da cidade, explicando a alta proporção de crianças no regime subsidiado de atenção em saúde e PPNA, entretanto, estas cifras evidenciaram redução significativa o último ano de medição.

É preciso continuar no fortalecimento das ações de prevenção das mortes previsíveis em crianças, considerando que a mortalidade infantil é resultado da complexa rede de determinantes em muitos níveis, sendo que as ações necessárias para salvar a vida das nossas crianças são conhecidas, constituindo-se no principal desafio a transferência do conhecimento à ação.

O pessoal da saúde, especialmente o de enfermagem, têm um papel fundamental na proteção da população infantil, através da intervenção nos determinantes sócias de saúde, tanto de senários de prestação de serviços de saúde clínicos e comunitários.

\section{Conflito de interesses e financiamento}

As pesquisadoras declaram não ter conflito de interesses.

Esta pesquisa foi financiada com recursos fornecidos pela Universidade de Antioquia.

\section{Bibliografía}

1. Organización Mundial de la Salud. Reducción de la mortalidad en niñez. [Internet]. 2018 [Consultado el 20 de septiembre de 2018]. Disponible en: http://www.who.int/es/news-room/fact-sheets/detail/ children-reducing-mortality

2. World Health Organization. Global Health Observatory (GHO) data. [Internet]. [ Consultado el 29 de julio de 2019]. Disponible en: http://www.who.int/gho/child health/mortality/mortality under five/en/

3. Programa de las Naciones Unidas para el Desarrollo. Objetivos de Desarrollo Sostenible. Objetivo 3: Salud y Bienestar. [Internet]. [Consultado el 13 de julio de 2018]. Disponible en: http://www.undp.org/ content/undp/es/home/sustainable-development-goals/goal-3-good-health-and-well-being.html\#targets

4. Centro Latinoamericano y Caribeño de Demografía (CELADE). Tasa de mortalidad infantil. Internet]. 2019 [Consultado el 30 de julio de 2019]. Disponible en: https://celade.cepal.org/redatam/pryesp/ cairo/WebHelp/Metalatina/tasa_de_mortalidad_infantil.htm 
ISSN-PRINT

$1794-9831$

E-ISSN 2322-7028

Vol. 17 No. 1

Ene - Abr 2020

Cúcuta, Colombia
5. Cardona $\mathrm{D}, \mathrm{A}$ costa $\mathrm{LD}$, Bertone $\mathrm{CL}$. Inequidades en salud entre países de Latinoamérica y el Caribe (2005-2010). Gac Sanit. [Internet]. 2013 [consultado 12 de diciembre de 2018]; (27) :292-297. Disponible en: http://dx.doi.org/10.1016/j.gaceta.2012.12.007

6. Departamento Administrativo Nacional de Estadísticas DANE. Resultados estimación tasa de mortalidad infantil. [Internet]. 2018 [Consultado el 23 de julio de 2018]. Disponible en: https://www.dane. gov.co/index.php/estadisticas-por-tema/demografia-y-poblacion/nacimientos-y-defunciones

7. Alcaldía de Medellín. Indicadores Básicos 2015. Situación de Salud de Medellín. [Internet]. 2015 [Consultado el 23 de julio de 2018]. Disponible en: https:/www.medellin.gov.co/irj/go/km/docs/ pccdesign/SubportaldelCiudadano_2/PlandeDesarrollo_0_19/IndicadoresyEstadsticas/Shared\%20 Content/Libros\%20de\%20indicadores/indicadores2015.pdf

8. Instituto Nacional de Salud. Observatorio Nacional de Salud. Tercer Informe ONS: Mortalidad evitable en Colombia para 1998-2011. [Internet]. 2014. Imprenta Nacional de Colombia, Bogotá, D.C., [Consultado el 13 de febrero de 2019]. Disponible en: https:/www.ins.gov.co/Direcciones/ONS/Informes/3.\%20Mortalidad\%20evitable.pdf

9. Jaramillo Mejía M, Chernichovsky D, Jiménez Moleón J. Determinantes de la mortalidad infantil en Colombia. Path Análisis. Rev. Salud Pública. [Internet]. 2018 [Consultado el 23 de septiembre de 2018]; 20(1): 3-9. Disponible en: http://www.scielo.org.co/scielo.php?script=sci arttext\&pid=S012400642018000100003\&lng=es\&nrm=iso\&tlng=es

10. Torres Melo J, Santander J. Introducción a las políticas públicas. Conceptos y herramientas desde la relación entre Estado y ciudadanía. Instituto de Estudios del Ministerio Público IEMP. [Internet]. 2013 [Consultado el 29 de julio de 2019] Disponible en: https://www.funcionpublica.gov.co/eva/ admon/files/empresas/ZW1wcmVzYV83Ng==/imgproductos/1450056996 ce38e6d218235ac89d6c8a14907a5a9c.pdf

11. Alcaldía de Medellín. Decreto 01277 de 2013, julio 5, por la cual se reglamenta el acuerdo 058 de 2011, mediante el cual se adopta la política pública de atención integral a la primera infancia Buen Comienzo, se desarrolló un sistema de atención integral y se modificó en acuerdo 14 de 2004. Gaceta oficial N 4176. [Internet]. Alcaldía de Medellín; 2013 [Consultado el 29 de julio de 2019]. Disponible en: https://www.medellin.gov.co/normograma/docs/d_alcamed_1277_2013.htm

12. Alcaldía de Medellín. Programa Buen Comienzo. Atención Integral a la primera infancia. [Internet]. Alcaldía de Medellín; 2010 [Consultado el 24 de julio de 2018]. Disponible en: https://web.oas. org/childhood/ES/Lists/Temas $\% 20 \% 20$ Proyectos $\% 20 \% 20$ Actividad $\% 20 \% 20$ Documento/Attachments $/ 366 / 3 \% 20$ Presentacion $\% 20$ Alcaldia $\% 20$ Medellin\%20Buen\%20Comienzo.pdf

13. Alcaldía de Medellín. Acuerdo 058, noviembre 30, por medio del cual se adopta la política pública de atención integral a la primera infancia Buen Comienzo, se desarrolla un sistema de atención integral y se modifica el acuerdo 14 de 2004. Gaceta oficial 3975. [Internet]. Alcaldía de Medellín; El Consejo, 2011. [Consultado el 29 de julio de 2019]. Disponible en: https://www.medellin.gov.co/normograma/ docs/a_conmed_0058_2011.htm

14. Alcaldía de Medellín. Resolución número 12760 de 2012, diciembre 4, por medio del cual se regula la prestación de servicios de atención integral a la primera infancia en desarrollo del programa Buen Comienzo. Medellín; Secretaria de Educación, 2012.

15. Cardona Sosa L, Medina C. Los efectos de los programas in utero en los resultados de los nacimientos: el caso de "Buen Comienzo" El efecto de los programas dirigidos a las gestoras en los indicadores de Nacer: El caso de "Buen Comienzo". Borradores de Economia [Internet]. 2016. Banco de la República de Colombia. [Consultado el 16 de febrero de 2019]. Disponible en: https://ideas.repec.org/p/bdr/borrec/955.html

16. Quintero MT, Álvarez LS, Góez JD. Evaluación del componente nutricional del programa Buen Comienzo de Medellín. Perspect Nut Hum. [Internet]. 2016 [Consultado 6 de noviembre de 2018]; 18(1):61-74. Disponible en: http://dx.doi.org/10.17533/udea.penh.v18n1a06 
17. República de Colombia. Ministerio de Salud. Resolución No 008430 de 1993, octubre 4, por la cual se establecen las normas científicas, técnicas y administrativas para la investigación en salud [Internet]. Santa Fe de Bogotá: El Ministerio; 1993 [consultado 13 de junio de 2018]. Disponible en: https://www. minsalud.gov.co/sites/rid/Lists/BibliotecaDigital/RIDE/DE/DIJ/RESOLUCION-8430-DE-1993.PDF

18. Maceira D. Centro de Estudios de Estado y Sociedad (CEDES), UNICEF. Estructura de los Programas de Salud en Niñez y Adolescencia en América Latina y el Caribe. Espacios Institucionales para alcanzar la Cobertura Universal en Salud. [Internet]. 2015 [Consultado 6 de diciembre de 2018]. Disponible en: http://www.cedes.org/publicaciones/documentos/Doc_t/Doc_t123.pdf

19. Quiroz KS, Pulgarín LM, Cardona D. Mortalidad de niños menores de 5 años después de la implementación de la estrategia atención integrada a las enfermedades prevalentes en la infancia (AIEPI) - Antioquia, Colombia 2002 - 2011. Rev Univ. Salud. [Internet]. 2017 [Consultado 6 de diciembre de 2018]; 17(2): 201-211. Disponible en: http://www.scielo.org.co/scielo.php?script=sci_abstract\&pi $\mathrm{d}=\mathrm{S} 0124-71072015000200006$

20. Augsburger AC, Gerlero S, Galende S, Moyano CB. La expresión de las desigualdades sociales en la mortalidad infantil. Información epidemiológica en regiones seleccionadas de la provincia de Santa Fe (Argentina). Rev. Fac. Nac. Salud Pública [Internet]. 2013 [Consultado 14 febrero de 2019]; 31(1):139-148. Disponible en: http://www.scielo.org.co/pdf/rfnsp/v31s1/v31s1a16.pdf

21. Comisión sobre determinantes sociales de la salud. Informe final de la comisión OMS sobre determinantes sociales de la salud. Subsanar las desigualdades en una generación: alcanzar la equidad sanitaria actuando sobre los determinantes sociales de la salud. 28 de agosto de 2008. [Internet]. [Consultado 15 febrero de 2019]. Disponible en: https://www.who.int/social_determinants/final_report/media/csdh_report_wrs_es.pdf

22. República de Colombia. Ley 100 de 1993, diciembre 23, Por la cual se crea el sistema de seguridad social integral y se dictan otras disposiciones. Diario Oficial No. 41.148. Bogotá: El Ministerio; 1993.

23. Secretaria Seccional de Salud y Protección Social de Antioquia. Análisis de situación de salud departamento de Antioquia Actualización 2017 [Internet]. 2017 [Consultado el 16 de febrero de 2019]. Disponible en: https://www.minsalud.gov.co/sites/rid/Lists/BibliotecaDigital/Forms/DispForm. aspx?ID $=16824$

24. Jaramillo MC, Chernichovsky D, Jiménez JJ. Brechas regionales de la mortalidad infantil en Colombia. Rev Peru Med Exp Salud Pública. [Internet]. 2013 [Consultado 6 de diciembre de 2018]; 30(4): 551-559. Disponible en: http://www.scielo.org.pe/pdf/rins/v30n4/a03v30n4.pdf

25. Jaramillo Mejía MC, Chernichovsky D, Jiménez Moleón J. Determinantes de la mortalidad infantil en Colombia. Path Análisis. Rev. salud pública [Internet]. 2018 [consultado 4 diciembre de 2018]; 20(1): 3-9. Disponible en: http://www.scielo.org.co/scielo.php?script=sci_arttext\&pid=S012400642018000100003\&lng=en

26. Rojas Gualdrón DF, Caicedo Velázquez B. Distancia al centro de atención en salud y mortalidad durante los primeros años de vida: revisión sistemática y metaanálisis. Rev. Fac. Nac. Salud Pública [Internet]. 2017 [consultado 15 de febrero de 2019]; 35(3):420-431. Disponible en: http://www.scielo. org.co/scielo.php?script=sci_arttext\&pid=S0120-386X2017000300420\&lng=en

27. Ezeh O, Agho K, Dibley M, Hall J, Page A. Risk factors for postneonatal, infant, child and under-5 mortality in Nigeria: a pooled cross-sectional analysis BMJ Open [Internet]. 2015 [consultado 15 de febrero de 2019]; 5(3). Disponible en: https://bmjopen.bmj.com/content/5/3/e006779

28. Leyton D, Valenzuela A. Trayectorias del cuidado de la salud infantil: El caso de la comunidad atacameña de toconao. Estud. atacam. [Internet]. 2017 [Consultado 15 de febrero de 2019]; 55:251-270. Disponible en https://scielo.conicyt.cl/scielo.php?script=sci_arttext\&pid=S0718$10432017000200013 \& \operatorname{lng}=$ es\&nrm $=$ iso

29. Ministerio de Salud y Protección Social. Análisis de Situación de Salud Colombia. [Internet]. 2017. 
ISSN-PRINT

1794-9831

E-ISSN 2322-7028

Vol. 17 No. 1

Ene - Abr 2020

Cúcuta, Colombia
[Consultado el 15 de febrero de 2019]. Disponible en: https:/www.minsalud.gov.co/sites/rid/Lists/ BibliotecaDigital/RIDE/VS/ED/PSP/asis-nacional-2017.pdf

30. Mogollón Pastrán SC, García Ubaque J. Tendencia y causas de la mortalidad infantil en municipios de frontera en Colombia, 2005-2011. Rev. Salud Pública. [Internet]. 2016 [consultado 4 octubre de 2018]; 18(5):700-713. Disponible en: http://www.scielo.org.co/scielo.php?script=sci_arttext\&pid=S012400642016000500700\&lng=es\&nrm=iso\&tlng=es

31. Comisión Económica para América Latina y el Caribe. Malnutrición en niños y niñas en América Latina y el Caribe. [Internet]. 2018 [Consultado 15 de febrero de 2019]. Disponible en: https://www. cepal.org/es/enfoques/malnutricion-ninos-ninas-america-latina-caribe

32. Andrade RD, Santos JS, Ambrosina Cardoso M, Iossi SMA, de La Ó Ramallo VM, Falleiros MD. Visita domiciliária: tecnologia de cuidado utilizada pelo enfermeiro na defesa da saúde da criança. Texto contexto - enferm. [Internet]. 2015 [consultado 16 de febrero de 2019]; 24(4): 1130-1136. Disponible en: http://www.scielo.br/scielo.php?script=sci_arttext\&pid=S0104-07072015000401130\&lng=en

33. Mosley W, Lincoln C. An Analytical Framework for the Study of Child Survival in Developing Countries. Population and Development Review. [Internet]. 1984 [Consultado 3 diciembre de 2018]; 10:2545. Disponible en: https://www.jstor.org/stable/2807954?origin=JSTOR-pdf\&seq=1\#page_scan_tab_ contents 